\title{
Nanophotonics
}

SPIEDigitalLibrary.org/jnp

\section{Fourier Modal Method and Its Applications in Computational Nanophotonics}

Chandraprakash Chindam 


\section{BOOK REVIEWS}

\section{Fourier Modal Method and Its Applications in Computational Nanophotonics}

Hwi Kim, Junghyun Park, and Byoungho Lee, 2012, ISBN-10: 1420088386, ISBN-13: 9781420088380, xii+313 pages, CRC Press, Boca Raton, \$179.95 hardcover, \$125 e-book. LCCN: 2011046920, OCLC:768728882

Reviewed by Chandraprakash Chindam, Pennsylvania State University, Department of Engineering Science and Mechanics, University Park, Pennsylvania 16802, USA

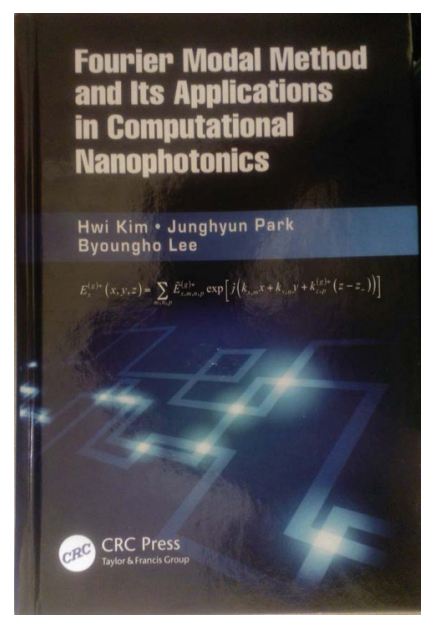

To develop research into practical applications, experimental results must be complemented with theory and computation. In the field of photonics, where the material medium is frequently inhomogeneous, the framework to solve Maxwell's equations with appropriate boundary conditions must be formulated. Kim, Park, and Lee establish this framework in Chapter 1 of Fourier Modal Method and Its Applications in Computational Nanophotonics. The remainder of this book is divided into six chapters.

Chapter 2 begins with the concepts of scattering matrix and Bloch eigenmodes for a single block - a one-dimensional slab of finite thickness. Here, the coupling coefficients used to determine the internal electromagnetic (EM) field and the intricacies in formulating the scattering matrix ( $\mathrm{S}$ matrix) method are explained. Although the parallel computation of the $\mathrm{S}$ matrix is mentioned, the authors do not show the methods for implementation even in the subsequent chapters. Chapter 2 then goes on to discuss the EM distribution in an assembly of one-dimensional blocks, called the N-block interconnection (or the multiblock). Here, the introduction of the partial multiblock seems unnecessary in contrast to what was mentioned in this section.

Based on the S-matrix formulation developed for a one-dimensional block, the use of the Fourier modal method (FMM) in studying two- and three-dimensional blocks is highlighted in Chapter 3. First, the S-matrix formulation for a one-dimensional block with periodicity in transversal permittivity and permeability (called the single block-structure) is developed. Next, the mathematical framework, similar to Chapter 2, is presented for different combinations of the finite-sized block and the semi-infinite spaces on either side of it. Additionally, the authors demonstrate an application of light propagation through apertures using the FMM. MATLAB codes are mentioned explicitly in different parts of the analysis to supplement understanding. This chapter provides a wholesome picture of the FMM and the scope of its applications.

Chapter 4 begins by presenting a progression of perfect-matched-layer (PML) methods for isolated geometries. In particular, implementations of the PML through absorbing boundary layers and through nonlinear coordinate transformations are discussed along with their limitations. This chapter concludes with examples of MATLAB implementations of the PML methods to study plasmonic lenses and plasmonic hot spots.

Chapter 5 extensively examines the local Fourier modal method (LFMM) used in identifying the Bloch eigenmodes of a superblock, which is a periodic assembly of multiblocks. As mentioned, this chapter deals with the application of FMM in nanophotonic devices. For instance, the authors discuss the propagation of a Bloch eigenmode in a photonic waveguide as well as the characteristics of one-directional-wave propagation in a photonic slab. Procedures that must be followed to implement the LFMM are first outlined and then analysis, similar to Chapters 2 and 3 , is extended to assemblies of superblocks. As wave propagation in a periodic medium (superblock assembly) is gaining keen interest currently, this chapter could have been more thorough if 
the LFMM had been presented with examples determining the dispersion relations for a threedimensionally nonhomogeneous, anisotropic, periodic media.

The focus of Chapter 6 is broad: networks of nanophotonic devices. Here, since the required concepts are already explained in previous chapters, first an algorithmic approach and then its implementation are presented. Some insights on the time scale of executing these seemingly cumbersome computational processes would have given the reader a sense of the complexities involved. Also, the current research directions towards the optimization of computation for investigating large nanophotonic networks could have benefited the graduate student.

Because this book is intended for graduate students, an inquisitive reader would wish for a more detailed bibliography. In particular, references on the implementation of PML methods for blocks of complicated shapes and references on the existing computing methods for circuits in Chapter 6 would have given the reader a more methodical understanding.

Despite the various shortcomings mentioned in this review, this book is perhaps the first of its kind in examining the computational aspects of nanophotonics and serves as a good reference for the research community. Altogether, Kim, Park, and Lee effectively present a focused analysis of current research in computational nanophotonics. 